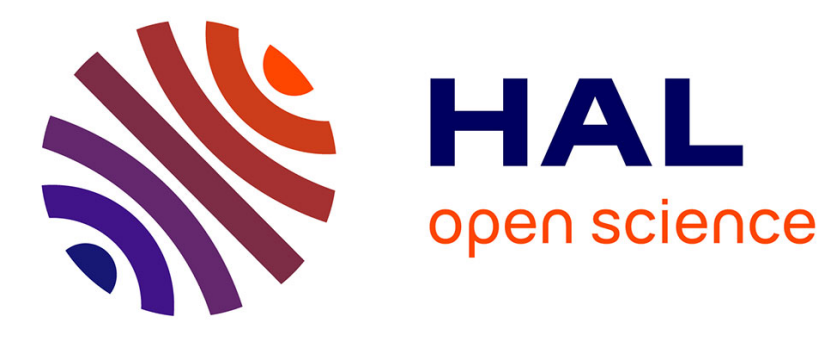

\title{
Chiromagnetoptics of Au and Ag Nanoparticulate Systems
}

Oleksander Kovalenko, Mircea Vomir, Bertrand Donnio, Jean-Louis L Gallani, Mircea V Rastei

\section{- To cite this version:}

Oleksander Kovalenko, Mircea Vomir, Bertrand Donnio, Jean-Louis L Gallani, Mircea V Rastei. Chiromagnetoptics of $\mathrm{Au}$ and Ag Nanoparticulate Systems. Journal of Physical Chemistry C, 2020, 124 (39), pp.21722-21729. 10.1021/acs.jpcc.0c06496 . hal-03404154

\section{HAL Id: hal-03404154 https://hal.science/hal-03404154}

Submitted on 26 Oct 2021

HAL is a multi-disciplinary open access archive for the deposit and dissemination of scientific research documents, whether they are published or not. The documents may come from teaching and research institutions in France or abroad, or from public or private research centers.
L'archive ouverte pluridisciplinaire HAL, est destinée au dépôt et à la diffusion de documents scientifiques de niveau recherche, publiés ou non, émanant des établissements d'enseignement et de recherche français ou étrangers, des laboratoires publics ou privés. 


\title{
Chiromagnetoptics of $\mathrm{Au}$ and $\mathrm{Ag}$ Nanoparticulate Systems
}

\author{
Oleksander Kovalenko, Mircea Vomir, Bertrand Donnio, Jean-Louis Gallani, Mircea V. Rastei*
}

Cite as J. Phys. Chem. C 2020, 124, 21722-21729

Publication Date: September 4, 2020

https://doi-org.inc.bib.cnrs.fr/10.1021/acs.jpcc.0c06496

\begin{abstract}
Photoelectron excitations into
$B=0+C P L+L S P$

$B \neq 0+C P L$

$B \neq 0+C P L+L S P$

unoccupied energy states are

fundamental in solid-state physics and many modern applications. The operation of such internal electronic transitions relies not only on photon absorption efficiency, which is pivotal in sensing, photovoltaics, active plasmonics, etc., but
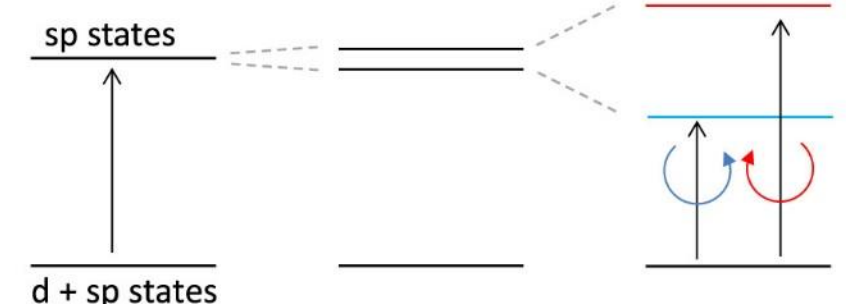

also on light-matter interactions and near/far-field spectroscopic techniques. Here, we report on a spectroscopic absorption fingerprint in magnetic circular dichroism (MCD) responses of various nanoparticulate systems. MCD spectra acquired at low temperatures on assemblies of uncoupled/coupled $\mathrm{Ag}$ and $\mathrm{Au}$ nanoparticles reveal asymmetric MCD spectral line shapes strongly depending on the nature and shape of nanoparticles and on their interparticular interactions. Assembling for instance the NPs into 2D networks with interparticle distances allowing dipolar interactions or transforming the spherical nanoparticles into disks was found to significantly alter the magneto-optic response in the spectral line shape. The findings are interpreted within the framework of chiral magneto-plasmonic effects by considering the $k$-dependent electronic states involved in both inter- and intraband electronic transitions.
\end{abstract}

\section{Introduction}

Plasmonic nanostructures have attracted great interest in the last 15 years due to their increasing potential for exceeding the diffraction limit in optics (1) and opening important perspectives in photonic applications. (2-5) At the heart of this research field is the ability of metallic nanostructures to temporarily store, confine, and release electromagnetic radiation. $(6,7)$ In this context, one sought after effect is the possibility of externally controlling this process. (8-10) To realize such functionalities, the application of an external magnetic field appears as a promising approach, (11-14) leading to magneto-optical (MO) effects. $(11,15,16)$ As a consequence, the recent advances in the research of plasmonic nanostructures continue to heighten magneto-optics, which has already been a blossoming field with respect to ferromagnetic (17-19) or semiconductor materials $\underline{(20-23)}$ for example. MO activity in non-ferromagnetic metals is nevertheless small, but the excitation of localized surface plasmon (LSP) dipoles can considerably enhance the magneto-optical coupling. $(24,25)$ This enhancement is the result of the Lorentz force, which exercises a torque on the plasmon dipoles inducing a far larger $\mathrm{MO}$ activity in metal nanostructures presenting LSP resonances than in their bulk or thin-film counterparts. $(25,26)$ MO activity associated with LSP resonances has also been found 
leading to linear and nonlinear responses at relatively low light intensities. $(27,28)$ Upon increasing the excitation densities, nonlinear effects become more important and a circularly polarized laser light at the localized surface plasmon (LSP) resonance may induce a current density proportional to the incident intensity of electric field and a net magnetic moment via the inverse Faraday effect. $(29,30)$

The Lorentz force is known to exert on moving charges exposed to magnetic fields. It is at the origin of many fundamental observations such as Hall and Zeeman effects but is also a key component for the development of advanced instrumentation such as cyclotron facilities or electron microscopy. For a charge moving at a fixed speed $v$, the Lorentz force reads as $\mathbf{F}=q(\mathbf{v} \times \mathbf{B})$. In the case of plasmonic excitations, the related oscillating dipole moment $\mathbf{p}(t)$ gives $(25) \mathbf{F}=\mathrm{d} \mathbf{p} / \mathbf{d} t \times \mathbf{B}$, and the associated energy may lift the degeneracy of electronic levels near the Fermi level (31) in a rather different way as in atomic systems. The difference resides in the fact that, in metals, the involved conduction levels form a continuous and $k$-dispersive sp-band, and the lift of the degeneracy is weak, barely observable by optical spectroscopy due to strong electron-electron correlations. When LSP excitations, induced by the oscillating electric field of the light, are associated with a large material polarisability, they can however significantly increase the unoccupied sp level splitting, facilitating the observation of a termA signal in MCD spectroscopy, which is by nature rather independent of temperature. (32)

When circular left- and right-handed light propagates in nanoparticulate plasmonic materials exposed to an external magnetic field, the electron excitations may be different on the spin-split levels because of quantum selection rules. This has been experimentally revealed through a specific MCD signature showing an asymmetric peak derivative-like spectrum. $(32,33)$ Furthermore, if a magnetic field splits the degenerate electronic levels of a nanoparticle by a quantity proportional to the field strength, the resulting magneto-plasmonic effect is maximal when the magnetic field is applied along the light propagation vector because the Lorentz force is perpendicular to the field. (34) In this picture, the importance of the nanometric size of the particles enters into play when considering the LSP modes. (35) Thus, the strong correlations between the electrons below the Fermi level are broken by the surface plasmon oscillations, as electrons are excited to the unoccupied states. This allows enough splitting of excited energy levels to induce an enhanced MCD signal with some asymmetry.

Electronic correlation effects were studied by Pineider et al. by analyzing in detail the asymmetry of the MCD spectra acquired on Au NPs. (33) There, it was quantitatively shown that interband transitions-being not part of plasma oscillations-impact the MCD signal by inducing a difference in left- and right-handed light absorption. As a result, the MCD signal appeared as an asymmetric doublepeak feature of the opposite sign. From a fundamental point of view, when such a spectral fingerprint depends on the onset of interband transitions, it might represent a unique spectral feature for characterizing many other nanometer-sized materials, which is thus very valuable for gaining information on their electronic properties. This is also because such spectra appear to depend on the size $(36)$ and shape $(37)$ of the NPs or their mutual interactions when they are coupled. $(38,39)$ These studies also demonstrate that MCD spectral line shape can reveal, at room temperature and low fields, the interplay between inter- and intraband transitions, which is central for spectra analyses. Another interesting study (40) has shown that magneto-optical effects can also be enhanced in natively magnetic Ni nanoparticles, particularly when they are organized into arrays or superlattices. All these examples suggest that magneto-optical activity might indeed be tailored to a good extent by a careful choice of the material nature, its internal structure, and/or organization. However, a study about the exact role played by the $k$-dependent electronic band distributions in the reciprocal space is still lacking, in particular when associated with spectra shifts, obstructing the access to additional information about the shape and orientation of nanoparticles. 
Here, we present an MCD study performed at $3 \mathrm{~K}$ and under magnetic fields of up to $8 \mathrm{~T}$ on both $\mathrm{Au}$ and $\mathrm{Ag}$ nanoparticles differing in sizes, shapes, and organization. The motivation of using Ag NPs is triggered by the fact that their interband transitions are far less intense at the LSP frequency than those of Au NPs. We also show that the aforementioned asymmetry of the MCD signal increases linearly with the applied field of up to $8 \mathrm{~T}$ and that it can be systematically tailored by altering the shape of the nanoparticles, as well as by assembling them in networks with controlled interparticle interactions. Besides the fundamental point of view, these findings can be of particular significance for the exploration of the orientation of NPs in networks and for sensing and metrology in general.

Experiments have been performed in high magnetic fields of up to $8 \mathrm{~T}$ with a home-build MCD setup, which includes an Oxford cryostat equipped with a superconducting magnet and optical accesses. This allows us to measure the evolution of the signal from low to high fields. All experiments presented here were carried out at $3 \mathrm{~K}$. MCD spectra have been obtained by measuring the MCD signal with the field parallel and antiparallel to the light propagation direction. The first set of experiments has been realized on spherical $\mathrm{Au}\left(d_{\mathrm{Au}}=8.7 \pm 0.5 \mathrm{~nm}\right)$ and $\mathrm{Ag}\left(d_{\mathrm{Ag}}=7.5 \pm 0.5 \mathrm{~nm}\right)$ NPs, which were dispersed in polyvinyl alcohol (PVA) polymer matrix, so that interparticle interactions can be neglected. The evolution of the MCD signal with the shape of the NPs has been obtained from experiments performed on Ag disks of 20-50 nm in diameter realized by anisotropic chemical etching of Ag NPs. The nanodisks were dispersed in a solution of PVA polymer and therefore were randomly oriented with respect to the substrate after drying of the film. The impact of interparticle interactions into the MCD signal was evidenced by performing experiments on few monolayers of Au NPs, where the distance between NPs was controlled by capping ligands, that is, oleylamine for $\mathrm{Au}$ and dodecanthiol for $\mathrm{Ag}$.

\section{Results and Discussion}

The role played by interband transitions and plasmonic intraband excitations in standard absorption spectroscopy is schematically shown in Figure 1. For fcc-crystallized noble metals, the first Brillouin zone (Figure 1a) contains an electronic band structure, which is known for bulk crystals, (41-43) especially close to Fermi energy (see Figure $1 \mathrm{c}, \mathrm{d}$ for $\mathrm{Au}$ ). Although these electronic states may slightly change in the case of nanoparticles, (44) they nevertheless represent a good starting point for understanding the electronic transitions upon light absorption. The particularity of Au and $\mathrm{Ag}$ metals is the partially occupied dispersive bands (6sp bands for Au and $5 \mathrm{sp}$ bands for $\mathrm{Ag}$ ) supporting free-like electrons, and the localized $d$-bands ( $5 d$ and $4 d$ bands for $A u$ and mostly $4 d$ band for $A g$ ), which are fully occupied, falling a few eV below the Fermi level. This electronic structure confers to these metals an interesting interplay between intraband and interband transitions, as the electrons from below the Fermi level can absorb visible light either by remaining within the dispersive band (intraband transitions) or by translating from occupied d-states to the first unoccupied states of the dispersive band (interband transitions), as schematically shown in Figure 1c,d. We stress here that both types of transitions occur along specific high-symmetry crystal directions, thus being very appealing for acquiring information about the shape of nanoparticles or for the development of characterization tools sensitive to NPs in networks, as discussed below.

The effect of inter- and intraband excitations on the absorption spectra of Au is schematized in Figure $\underline{1 b}$, with the overall response (black line) depicted as a sum of LSP resonance (intraband collective excitations) and interband transitions. It is worth noting that in the visible spectral range, interband transitions are expected to first occur along the $X-\Gamma$ direction, at a location labeled $\Delta x$, where one dispersive sp-band intersects the Fermi level. For $\mathrm{Au}$, this location falls around $1.7 \mathrm{eV}(730 \mathrm{~nm})$ where the absorption spectra start showing the first signs of an increase in signal, as illustrated by the red 
line in Figure 1b. Increasing the photon energy, electrons from the d-bands can then be excited upper in the unoccupied sp-band (not shown), increasing even more the absorption, also because of the flatness of the band at the $X$-point. Above $\sim 2.4 \mathrm{eV}(516 \mathrm{~nm})$, another interband transition $\Delta_{L}$ starts playing a role in the absorption spectra of $A u$, being localized in the $L-W$ direction. These two effects explain the progressive but significant increase of absorption when approaching the UV spectral range (red line in Figure 1b) for bulk Au.

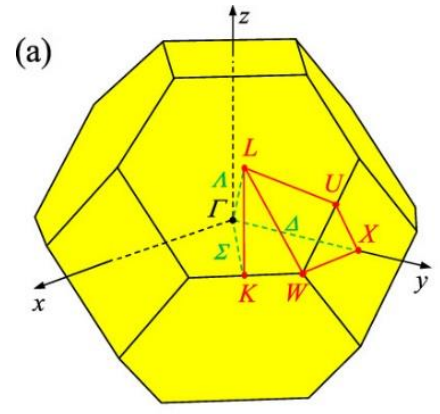

(c)

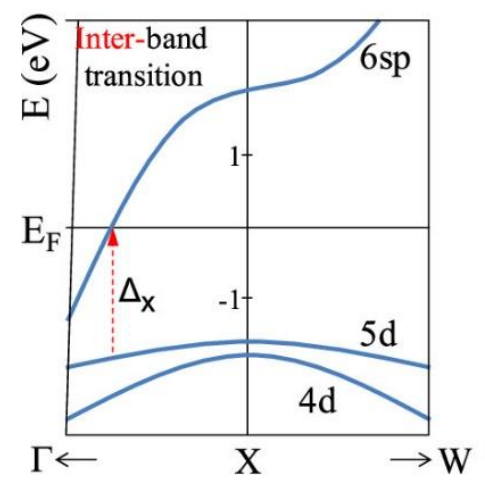

(b)

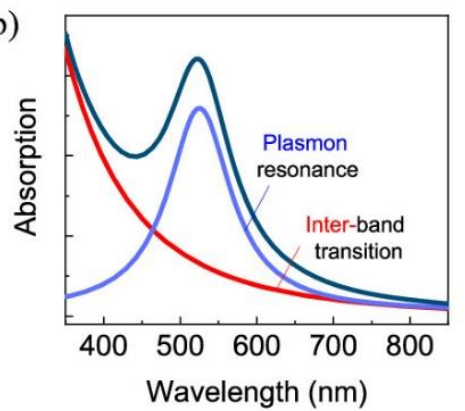

(d)

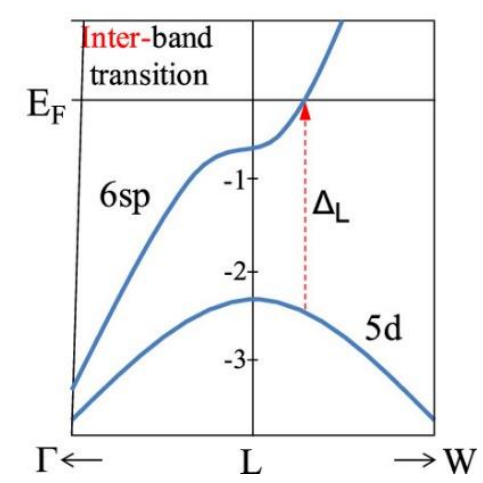

Figure 1. Band structure and electronic excitations upon absorption of light. (a) First Brillouin zone of fcc-Au showing the high-symmetry crystal directions. (b) Schematic of absorption spectra resulting from plasmon (intraband) resonance and interband contributions. (c, d) Band structure near $X$ and $L$ points revealing $\Delta_{x}$ and $\Delta_{L}$ thresholds for interband transitions falling in the visible range.

For bulk Ag, the shape of the electronic band structure is very similar to that of $A u$. The difference resides in the fact that the absorption due to interband transitions raises at higher energies, typically above $4.0 \mathrm{eV}(\sim 310 \mathrm{~nm})$. In other words, the two interband transition thresholds ( $\Delta_{x}$ and $\left.\Delta_{L}\right)$ in $\mathrm{Ag}$ are roughly at energies $2.0 \mathrm{eV}$ higher, that is, the $\mathrm{d}$ - and sp-bands near $X$ and $L$ points are more separated in energy. This is now a well-known band structure property of bulk Au and Ag discussed in several experimental (45-48) and theoretical works. (49-53)

In the case of NPs made from these metals, visible light can also be absorbed by LSP excitations arising from collective oscillations $\underline{(54,55)}$ of the electrons within the sp conduction bands (intraband excitations). (56-58) Also note that the difference in the LSP resonance modes for spherical Au and Ag NPs is only about $0.62 \mathrm{eV}$ (Au: $\sim 2.33 \mathrm{eV}$ and Ag: $\sim 2.95 \mathrm{eV}$ ). Altogether, these electronic phenomena discussed here in the context of standard absorption spectroscopy in the visible range constitute the essential background to interpret the MCD spectra described hereafter.

\subsection{Noninteracting Au and Ag NPs}

In Figure 2, we show the MCD spectra for Au and Ag NPs acquired at $3 \mathrm{~K}$ for increasing values of the magnetic field. As expected, it is seen in both cases that the spectra display a positive and a negative 
peak, with a crossing at around $530 \mathrm{~nm}$ for Au and at $420 \mathrm{~nm}$ for Ag. These values given here by the positions where the signal crosses zero correspond well to the known energies of the LPS resonances of Au and Ag NPs.

As seen, besides the spectral positions, another difference between $\mathrm{Au}$ and $\mathrm{Ag}$ spectra is the asymmetry between the amplitudes of the two peaks, which is critically larger for Au than for Ag. The low-wavelength (positive) peak is however smaller in both cases, which is therefore a common characteristic of Au and Ag. As first suggested in ref (32) for Au NPs, this double-peak shape in MCD spectra arises from the Zeeman splitting of the dispersive sp-bands by the external magnetic field, as sketched in the inset of Figure 2. a Left and right circularly polarized light are thus differently absorbed on the two spin-split levels because of selection rules. Equal amplitudes of the double-peak resonance would be expected if only sp-bands splitting is considered. The asymmetry of the peak amplitudes is consequently an additional effect, which cannot be explained through the quantum selection rules alone.

(a)

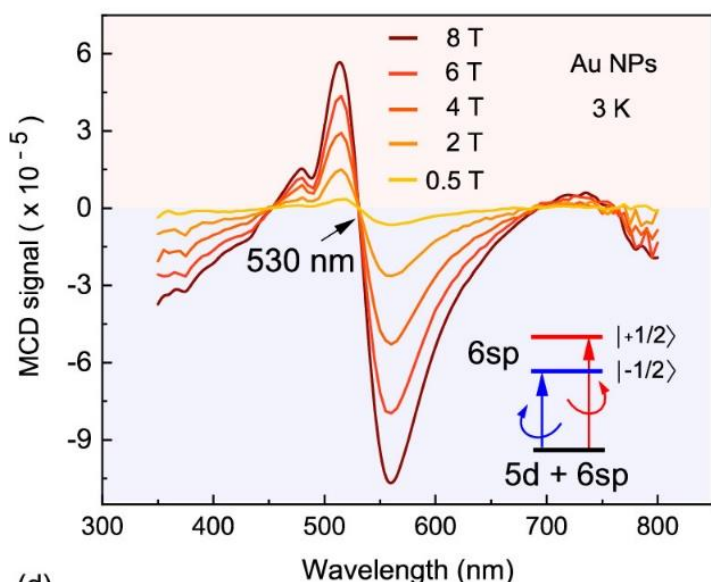

(d)

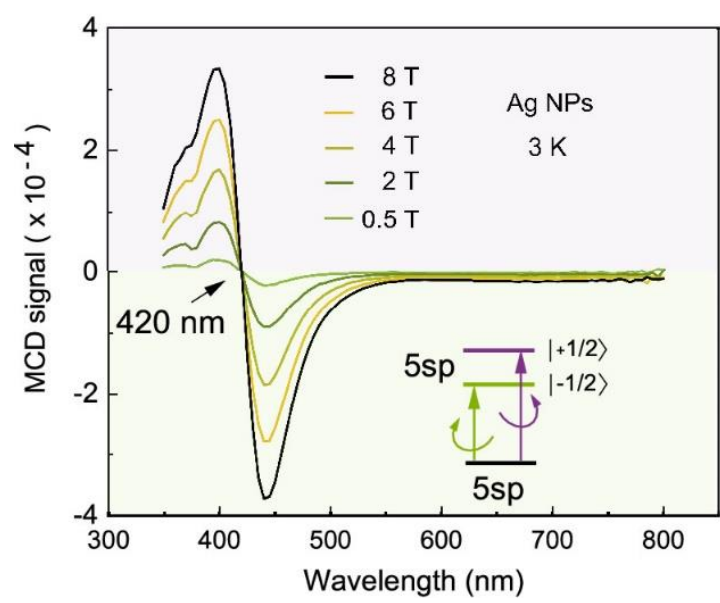

(b)

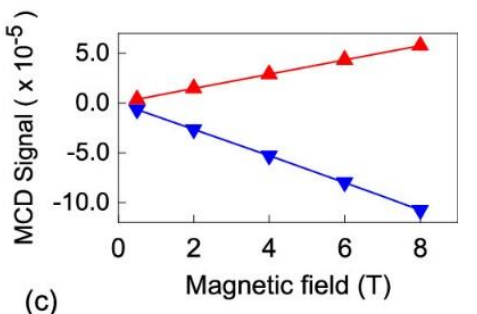

(c)

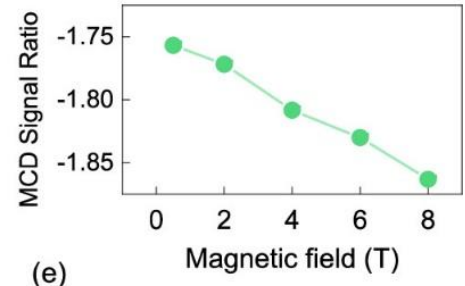

(e)

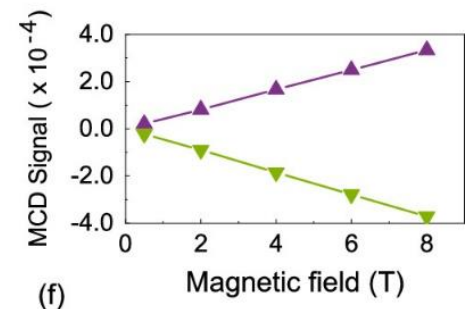

(f)

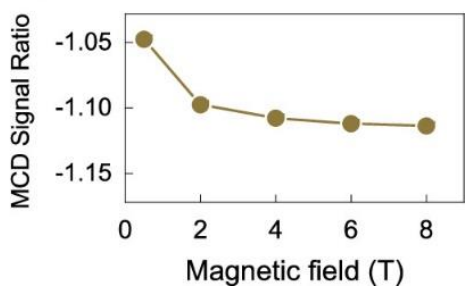

Figure 2. MCD characterization of noninteracting Au and Ag spherical NPs. (a, d) Magnetic field dependence of $\mathrm{MCD}$ spectra at $3 \mathrm{~K}$ for $\mathrm{Au}$ and $\mathrm{Ag}$, respectively. Intensities of positive and negative peaks plotted as a function of the magnetic field for (b) Au and (e) Ag. Signal ratio obtained by dividing the intensity of the negative peak by the intensity of the positive peak for $\mathrm{Au}$ (c) and $\mathrm{Ag}$ (f).

The variation of the amplitudes of the peaks with the magnetic field is shown in Figure $2 b, d$ along with the resulting asymmetry (Figure $2 c, f$ ) defined as the amplitude ratio of negative and positive peaks. It 
is seen that both $\mathrm{Au}$ and $\mathrm{Ag}$ show a linear increase in the amplitude of the peaks with the magnetic field.

The asymmetry increases almost linearly with the field for $\mathrm{Au}$ (Figure $2 \mathrm{c}$ ), remaining on the contrary rather constant around 1.1 for $\mathrm{Ag}$ (Figure 2f). Following Pineider et al., (33) this suggests that interband transitions impact to a larger extent the MCD spectra for Au than for Ag NPs. In fact, our comparative study on Au and Ag NPs can be seen here as a direct experimental demonstration of the model introduced by Pineider et al. (33) The inter- and intraband electronic transitions are known to impact on the dielectric tensor of metallic nanoparticles subjected to a magnetic field, (59) as considered in Section 2.3 for the modeling of the magneto-optical response.

\subsection{Two-Dimensional Networks of Au NPs}

In Figure 3, we show the MCD spectra for Au NPs organized in a hexagonal network. The interaction between the NPs was tailored by the capping organic ligands, whose length ensures a plasmonic coupling between first-order neighbors. Such interparticle interactions are known to slightly shift the energy of the LSP mode toward lower energies (red shift). $(60,61)$ As the interband transitions are based on the internal crystal structure, they should not be affected much; one can therefore expect a reduction in the measured asymmetry as compared with isolated NPs (Figure 2). This is because the LSP mode falls at larger wavelengths, away from the energies where interband excitations occur.

(a)

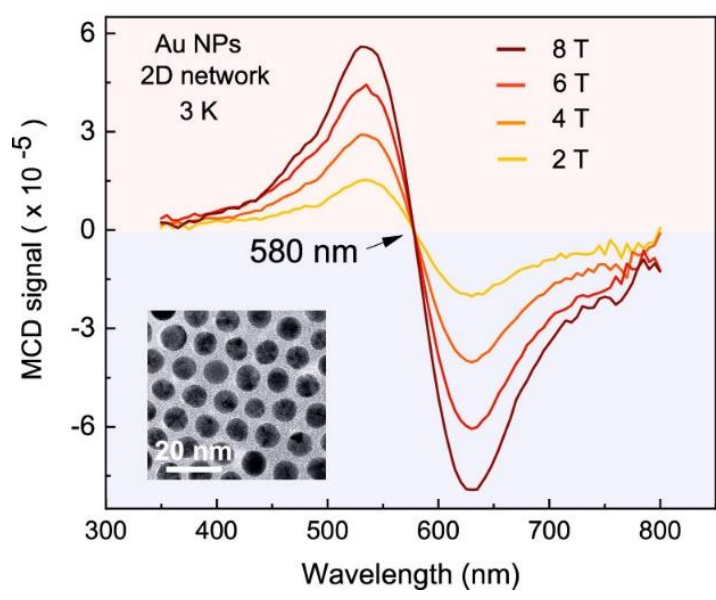

(b)
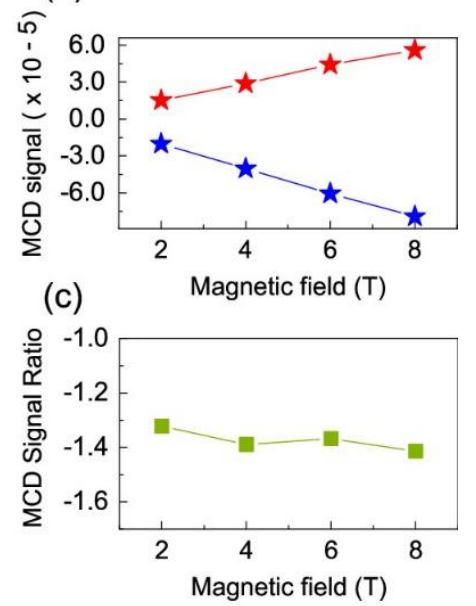

Figure 3. MCD characterization of interacting Au NPs. (a) Magnetic field dependence of MCD spectra at $3 \mathrm{~K}$ for $\mathrm{Au}$ NPs assembled in a hexagonal lattice. Inset is an electron microscopy image. (b) Intensities of positive and negative peaks as a function of the magnetic field for the red-shifted LSP mode $(580 \mathrm{~m})$. (c) Signal ratio obtained by dividing the intensity of the negative peak by the intensity of the positive peak, revealing a reduced asymmetry, as compared to noninteracting Au NPs (see Figure 1a,c,d), effect induced by the interparticle coupling.

The data on interacting NPs from Figure 3 is therefore in a full agreement with the description made above for isolated NPs, again indicating that the asymmetry of the MCD spectra is based on the interplay between interband and intraband transitions.

\subsection{Modeling the Magneto-Optical Response}

To provide a theoretical description of the experimental observations presented above, we use the effective medium approximations (EMA) to obtain the reflectivity (and absorption) of the NPs assembly and the Voigt theory to account for the magneto-optic response. We first use the Maxwell-

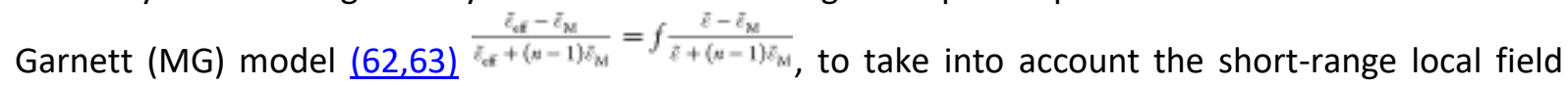




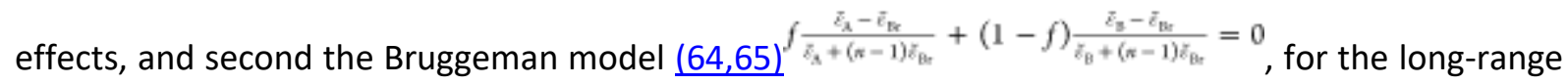
dipolar interaction. For the MG model, $\tilde{\varepsilon}_{\text {eff }}$ is the effective dielectric constant, $\tilde{\varepsilon}_{\mathrm{M}}$ is the dielectric constant of the matrix (MG model), and $\tilde{\varepsilon}$ is the dielectric constant of the metal. For the Bruggeman model, $\tilde{\varepsilon}_{A}$ and $\tilde{\varepsilon}_{B}$ are the dielectric functions of the matrix and metal and $\tilde{\varepsilon}_{B r}$ is the effective dielectric constant of the medium. In both the models, $f$ is the volume fraction of the metal.
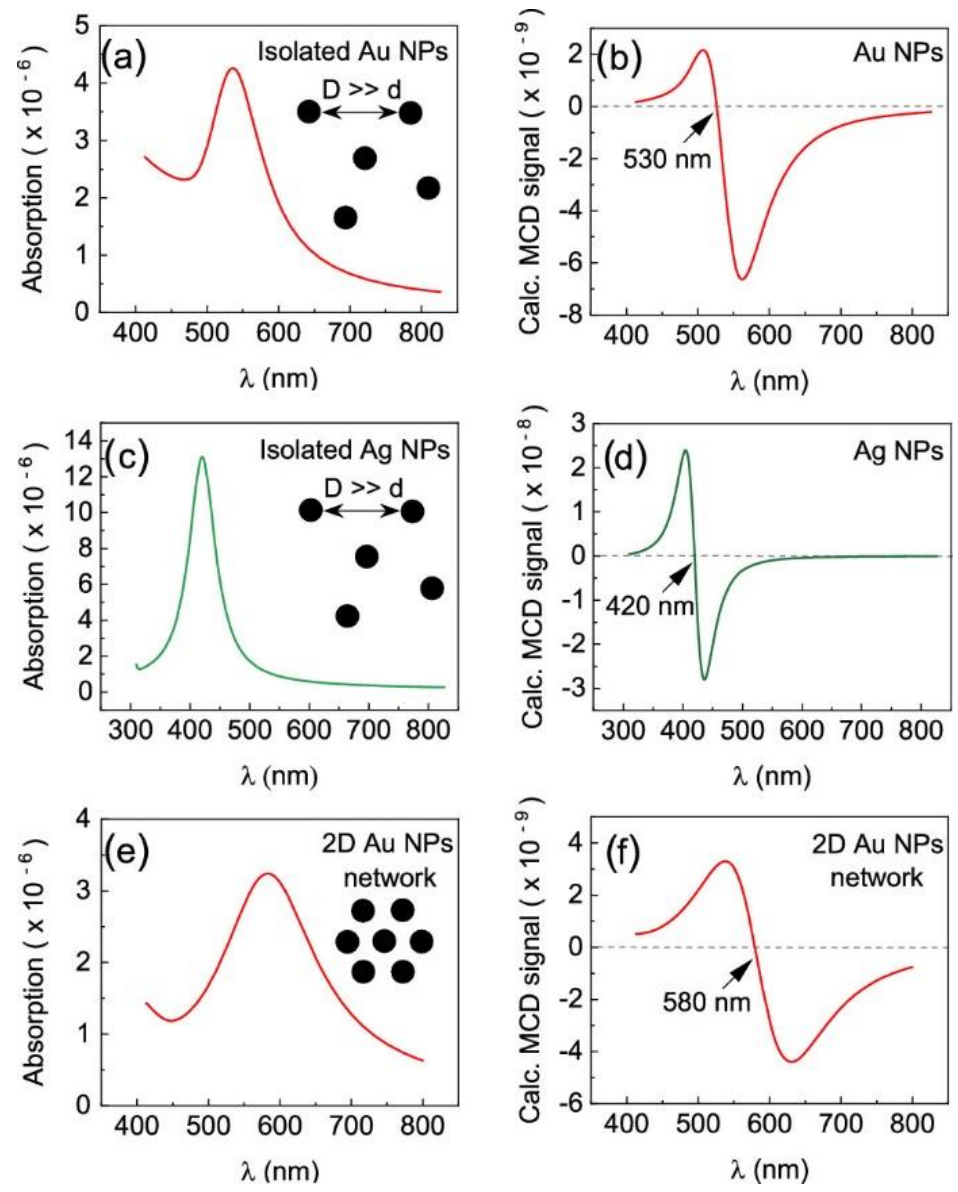

Figure 4. Calculated absorption (a, c, e) and $\operatorname{MCD}(b, d, f)$ responses of noninteracting (a-d) and interacting (e, f) NPs. Calculations were performed using the MG and Bruggeman models, as well as the Voigt formalism.

The bulk metal $(\mathrm{Au})$ dielectric function is considered as consisting of two parts. The first one is related to the intraband transitions described by the Drude model ${ }^{\left.\tilde{f}_{\mathrm{f}}(\omega)=1-\frac{e_{f}^{2}}{\omega(\omega+\mathrm{i})}\right)}$, where $\omega_{\mathrm{p}}$ is the plasmon frequency, $\gamma$ is the free-electron damping, and $\hbar \omega$ is the photon energy. The second contribution in the dielectric function is based on the interband excitations (Lindhard RPA theory) $\tilde{E}_{b}(\omega)=2 K \int_{E_{g}}^{\infty} \mathrm{d} E \frac{\sqrt{E-E_{g}}}{E}[1-F(E, T)] \frac{1}{[\hbar \omega]^{3}-E^{2}-(h \alpha)^{2}-2 i \hbar \omega h \alpha}$, where, $E, F, \hbar \alpha, k_{\mathrm{B}} T, E_{\mathrm{f}}$, and $E_{\mathrm{g}}$ are the interband energy, Fermi distribution function, damping constant, thermal energy, Fermi energy, and band gap energy, respectively. $K$ is a constant parameter, which depends on the transition dipole moment and electron effective mass. These formulations are known to approximate well the effective response (and hence the optical absorption signal) of composite media comprising noninteracting metal NPs. $\underline{(66,67)}$ 
Finally, the Voigt model (68) was used for the calculation of the nondiagonal components of the dielectric tensor $\tilde{\varepsilon}$ from which the time-dependent magnetic signal can be obtained $\tilde{\varepsilon}_{x z} \approx \tilde{\varepsilon}_{y y} \approx \tilde{\varepsilon}_{z z} \approx \tilde{E}(\omega), \varepsilon_{x y} \approx \frac{\omega_{p}^{t} \omega_{i}}{\omega\left[(\omega+t h)^{2}-\omega_{t}^{1} 1\right.}$, leading to an estimation of the MCD signal.

The calculated absorption and magnetic signals for noninteracting Au and Ag NPs are shown in Figure $4 a-d$ and are in good agreement with the experimental results. Both the peak positions and the asymmetry are obtained using typical parameters for $\mathrm{Au}$ and $\mathrm{Ag}$. These analytical models therefore represent an essential base for the emergence of simple fitting models, which could be used systematically for extracting parameters featuring the behavior of the isolated or coupled NPs.

\subsection{The Role of the Shape: Ag Nanodisks}

Given what has been said above for spherical noninteracting NPs, let us now imagine how changes in the plasmonic modes arising from shape modifications affect the interband transitions, since the collective resonances will fall at different energies. In contrast, the intraband transitions, strictly related to the band structures, are in turn expected to stay rather unaffected by the shape of the crystals. To gain additional insights into these electronic excitation phenomena, we performed MCD experiments on Ag nanodisks obtained by the chemical anisotropic etching of spherical Ag NPs. This shape modification is indeed expected to impact the LSP modes, 169,70$)$ while the electronic band structure is not likely to change significantly.

The MCD spectra of the noninteracting Ag nanodisks are shown in Figure 5. As expected, the "disk" shape brings an additional spectral feature in the visible range, at about $620 \mathrm{~nm}$. This feature is assigned to the longitudinal LSP plasmonic mode falling now at lower energy than the transversal LSP mode, which remains at $420 \mathrm{~nm}$, as for spherical NPs. The broadness of the MCD spectra feature at $620 \mathrm{~nm}$ is probably induced by the large lateral size distribution of the nanodisks, which as seen in the inset of Figure $5 \mathrm{a}$ is relatively large in our samples. This agrees with extinction spectra (not shown) which revealed a broad line shape at the respective energy (FWHM $\sim 150 \mathrm{~nm}$ ). Interestingly, the asymmetry of the MCD spectral feature at $420 \mathrm{~nm}$ displays now a significant enhancement as compared to spherical Ag NPs, increasing up to a ratio of 20 . While several factors can participate in such a modification in the asymmetry, including absorption changes and/or multipolar plasmonic effects, we restrain our interpretation of $k$-dependent band structure effects, as introduced in Figure $\underline{1 .}$

(a)

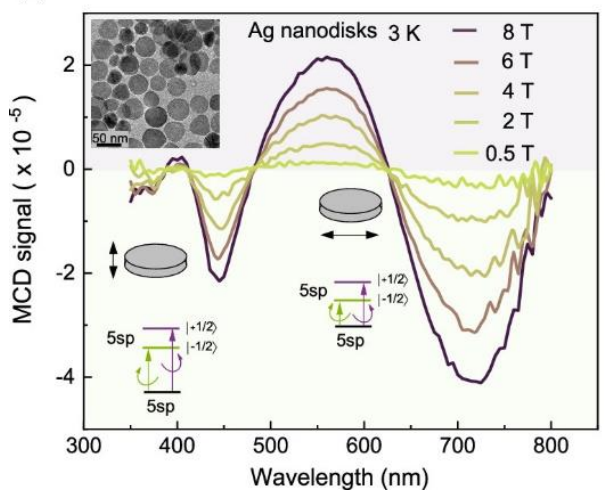

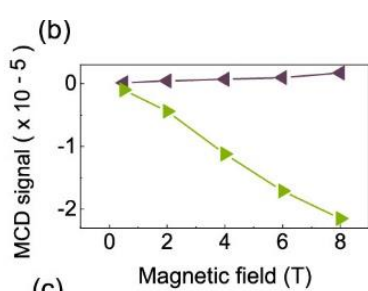

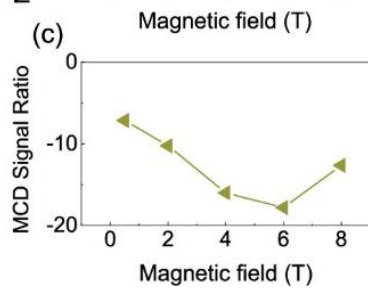

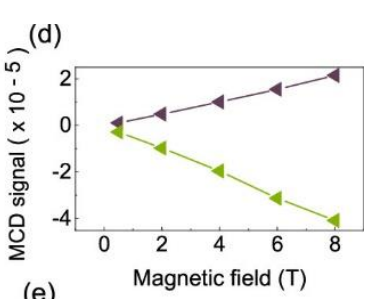

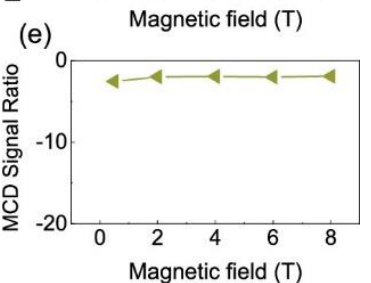

Figure 5. MCD characterization of noninteracting Ag nanodisks. (a) Magnetic field dependence of MCD spectra at $3 \mathrm{~K}$ for Ag disks. Inset: electron microscopy image showing Ag nanodisks drop-cast on a microscope grid. (b, d) Intensities of positive and negative peaks as a function of the magnetic field for the transversal LSP mode (420 
$\mathrm{m})$ and for the longitudinal LSP mode $(620 \mathrm{~nm})$, respectively. Signal ratio obtained by dividing the intensity of the negative peak by the intensity of the positive peak for (c) transversal LSP mode and (e) longitudinal LSP mode.

\section{5. $k$-Dependent Effects}

The MCD signal of noninteracting Au and Ag spherical NPs increases linearly with the magnetic field up to $8 \mathrm{~T}$, as a consequence of the dipolar origin of plasmon excitations and of band splitting. The magneto-plasmonic MCD peak asymmetry of Ag NPs is significantly smaller than that of Au NPs, demonstrating, as proposed by Pineider et al., (33) that the interband transitions are indeed responsible for the MCD asymmetric peak signals. In the light of these experimental findings and descriptions on noninteracting NPs, the spectra of nanodisks suggest that either the interband transitions are significantly enhanced or the intraband excitations in the $k$-dispersive $s p$-band are reduced. To interpret these experimental findings, we consider the anisotropic chemical etching of fcc nanoparticles, which in our case preferentially occurs along one of the less-compact crystal faces, that is, $(100)$ crystal planes. $(71,72)$ The band structure of $\mathrm{Ag}$, which as stated above is qualitatively very similar to that of $A u$, has the $X-\Gamma$ and $L-W$ high-symmetry directions related to the (100) crystallographic planes (Figure 1a). When the chemical etching of Ag spheres produces nanodisks with two large (100) faces, the longitudinal LSP mode is oriented along (100), while the transversal LSP mode is oscillating perpendicularly. Looking at the first Brillouin zone (Figure 1a), it can be deduced that the sp-band that holds collective oscillations of electrons with a wavevector $k$ perpendicular to the (100) plane is the one laying along the $X-\Gamma$ direction. In contrast, electrons within the sp-band corresponding to the $L-W$ direction are not expected to efficiently participate in this transversal LSP resonant mode. Again, it is worth noting that interband transitions are likely to remain unaffected by the shape of the NPs. The giant enhancement of asymmetry observed in Figure 5 therefore speaks in favor of weak intraband transitions in the dispersive sp-band along the $X-\Gamma$ direction. In the case of nanodisks with preferentially (111) faces, the transverse LSP mode $(420 \mathrm{~nm})$ would correspond to intraband transitions along the $L-\Gamma$ direction where, as observed in Figure $1 \mathrm{~d}$, no empty states are available. The low absorption due to the transverse LSP mode originates mainly from the weak component in the $X-$ $\Gamma$ direction. This results in a low MCD signal amplitude and a high asymmetry, as observed in Figure 5a. Whether this finding holds true for spherical NPs as well remains an interesting question, which might be of interest to be addressed in further work, by means of photoemission spectroscopy studies for example. It is also interesting to remark that the different effective masses of electrons in the two dispersive bands may play a role, since quasiparticle corrections are known to impact the photoelectron excitations. $(47,73)$ Moreover, the fact that there is an abrupt change in the $k$ wavevector at the $X$-point in the unoccupied part of the Brillouin zone can also participate to inefficient intraband transitions in this region of the reciprocal space. It can nevertheless be stated that the shapeinduced symmetry breaking that separates in energy the two LSP modes combined with MCD spectroscopy thus represents a very attractive technique to explore band structure effects in relation to photoelectron excitations. This is because the asymmetry of the MCD signal is linked to the balance between the inter- and intraband electron excitations. These findings indicate that MDC spectroscopy can be a very powerful tool for characterization of the shape of the NPs, as well as for the investigation of the $k$-dependent electronic states upon production of chemically synthesized NPs.

\section{Conclusions}

The MCD signal of noninteracting Au and Ag spherical NPs, featured by an asymmetric double-peak shape, has a linear dependence on the magnetic field. This particular line shape can be utilized as a 
spectroscopic fingerprint informing about NPs nature or shape, as well as their mutual interactions when assembled in networks. Indeed, for a disk shape, the MCD spectra revealed that the plasmon resonance, typical for spherical NPs, falling at $420 \mathrm{~nm}$ is preserved, constituting the transversal LSP resonance mode. In turn, the MCD asymmetry presents a giant enhancement when compared with spherical Ag NPs. These results were interpreted by analyzing the electronic band structure of the fcccrystalline lattice where both dispersive bands-supporting collective excitations of electrons-and localized bands-promoting interband transitions-are present close to the Fermi level. We have also shown that the organization of Au NPs in 2D hexagonal networks, which favor interparticle interactions, induces a red shift of the plasmon resonance frequency. As a consequence, the asymmetry of the MCD peaks is reduced, demonstrating that the interplay between intra- and interband transitions are responsible for the asymmetry. Overall, we propose here that the asymmetry of the MCD spectral features is a very powerful contrivance for studying structure-, shape-, or interaction-property relationships. Besides the fundamental point of view, these findings might be of great importance for deciphering electron-level contribution in light-matter interaction, analytical chemistry, sensing, or in the sturdily promising field of plasmon-based applications.

\section{Author Information}

Corresponding Author: M. V. Rastei - Institut de Physique et Chimie des Matériaux de Strasbourg, CNRS, Université de Strasbourg, F-67034 Strasbourg, France; http://orcid.org/0000-0003-3927-1409; Email: rastei@ipcms.unistra.fr. Authors: O. Kovalenko - Institut de Physique et Chimie des Matériaux de Strasbourg, CNRS, Université de Strasbourg, F-67034 Strasbourg, France; M. Vomir - Institut de Physique et Chimie des Matériaux de Strasbourg, CNRS, Université de Strasbourg, F-67034 Strasbourg, France; B. Donnio - Institut de Physique et Chimie des Matériaux de Strasbourg, CNRS, Université de Strasbourg, F-67034 Strasbourg, France; http://orcid.org/0000-0001-5907-7705; J. L. Gallani - Institut de Physique et Chimie des Matériaux de Strasbourg, CNRS, Université de Strasbourg, F-67034 Strasbourg, France

Notes: The authors declare no competing financial interest.

\section{Acknowledgement}

We thank Prof. Franck Neese and Dr. Bill Eckhard from MPI Mülheim for the preliminary tests on some of our samples and N. Beyer (IPCMS) for technical assistance. This work is supported by ANRs OHRISQUE SMARAGD (14 OHRI 0008 01) and partially by EOARD (FA8655-13-1-3001) and EquipEx UNION No. ANR-10-EQPX-52.

Additional Note : Our MCD spectra as a function of temperature (3-300 K) indeed indicate that a Cterm signal involving the splitting of the ground states is not important here.

\section{References}

$1 \underline{1}$ Barnes, W. L.; Dereux, A.; Ebbesen, T. W. Surface plasmon subwavelength optics. Nature 2003, 424, 824-830, DOI: 10.1038/nature01937 [Crossref], [PubMed], [CAS], Google Scholar

2. Genet, C.; Ebbesen, T. W. Light in Tiny Holes. In Nanoscience and Technology; Macmillan Publishers Ltd: U.K., 2009; pp 205- 212 [Crossref], Google Scholar 
3. Chen, H.-T.; Taylor, A. J.; Yu, N. A review of metasurfaces: physics and applications. Rep. Prog. Phys. 2016, 79, 076401 DOI: 10.1088/0034-4885/79/7/076401 [Crossref], [PubMed], [CAS], Google Scholar 4. Lustig, W. P. Metal-organic frameworks: functional luminescent and photonic materials for sensing applications. Chem. Soc. Rev. 2017, 46, 3242-3285, DOI: 10.1039/C6CS00930A [Crossref], [PubMed], [CAS], Google Scholar

5. Gramotnev, D. K.; Bozhevolnyi, S. I. Plasmonics beyond the diffraction limit. Nat. Photonics 2010, 4, 83-91, DOI: 10.1038/nphoton.2009.282 [Crossref], [CAS], Google Scholar

6. Liu, H. Magnetic plasmon hybridization and optical activity at optical frequencies in metallic nanostructures. Phys. Rev. B 2007, 76, 073101 DOI: 10.1103/PhysRevB.76.073101 [Crossref], [CAS], Google Scholar

7. Gramotnev, D. K.; Bozhevolnyi, S. I. Nanofocusing of electromagnetic radiation. Nat. Photonics 2014, 8, 13- 22, DOI: 10.1038/nphoton.2013.232 [Crossref], [CAS], Google Scholar

8. Krasavin, A. V.; Zheludev, N. I. Active plasmonics: Controlling signals in Au/Ga waveguide using nanoscale structural transformations. Appl. Phys. Lett. 2004, 84, 1416-1418, DOI: 10.1063/1.1650904 [Crossref], [CAS], Google Scholar

9. Pala, R. A.; Shimizu, K. T.; Melosh, N. A.; Brongersma, M. L. A Nonvolatile Plasmonic Switch Employing Photochromic Molecules. Nano Lett. 2008, 8, 1506- 1510, DOI: 10.1021/nl0808839 [ACS Full Text ], [CAS], Google Scholar

10. Maoz, B. M. Plasmonic chiroptical response of silver nanoparticles interacting with chiral supramolecular assemblies. J. Am. Chem. Soc. 2012, 134, 17807-17813, DOI: 10.1021/ja309016k [ACS Full Text ], [CAS], Google Scholar

11. Temnov, V. V. Active magneto-plasmonics in hybrid metal-ferromagnet structures. Nat. Photonics 2010, 4, 107- 111, DOI: 10.1038/nphoton.2009.265 [Crossref], [CAS], Google Scholar

12. González-Díaz, J. B. Plasmonic Au/Co/Au Nanosandwiches with Enhanced Magneto-optical Activity. Small 2008, 4, 202- 205, DOI: 10.1002/smll.200700594 [Crossref], [PubMed], [CAS], Google Scholar 13. Armelles, G. Magnetic field modulation of chirooptical effects in magnetoplasmonic structures. Nanoscale 2014, 6, 3737- 3741, DOI: 10.1039/c3nr05889a [Crossref], [PubMed], [CAS], Google $\underline{\text { Scholar }}$

14. Qin, J. Switching the Optical Chirality in Magnetoplasmonic Metasurfaces Using Applied Magnetic Fields. ACS Nano 2020, 14, 2808-2816, DOI: 10.1021/acsnano.9b05062 [ACS Full Text], [CAS], Google $\underline{\text { Scholar }}$

15. Rikken, G. L. J. A.; Raupach, E. Observation of magneto-chiral dichroism. Nature 1997, 390, $493-$ 494, DOI: $10.1038 / 37323$ [Crossref], [CAS], Google Scholar

16. Armelles, G. Magnetoplasmonic nanostructures: systems supporting both plasmonic and magnetic properties. J. Opt. A: Pure Appl. Opt. 2009, 11, 114023 DOI: 10.1088/1464-4258/11/11/114023 [Crossref], [CAS], Google Scholar

17. Moog, E. R.; Liu, C.; Bader, S. D.; Zak, J. Thickness and polarization dependence of the magnetooptic signal from ultrathin ferromagnetic films. Phys. Rev. B 1989, 39, 6949- 6956, DOI: 10.1103/PhysRevB.39.6949 [Crossref], [PubMed], [CAS], Google Scholar

18. Buschow, K. H. J.; van Engen, P. G.; Jongebreur, R. Magneto-optical properties of metallic ferromagnetic materials. J. Magn. Magn. Mater. 1983, 38, 1- 22, DOI: 10.1016/0304-8853(83)900975 [Crossref], [CAS], Google Scholar

19. Gridnev, V. N.; Pavlov, V. V.; Pisarev, R. V.; Kirilyuk, A.; Rasing, T. Second harmonic generation in anisotropic magnetic films. Phys. Rev. B 2001, 63, 184407 DOI: 10.1103/PhysRevB.63.184407 [Crossref], [CAS], Google Scholar

20. Brion, J. J.; Wallis, R. F.; Hartstein, A.; Burstein, E. Theory of Surface Magnetoplasmons in Semiconductors. Phys. Rev. Lett. 1972, 28, 1455- 1458, DOI: 10.1103/PhysRevLett.28.1455 [Crossref], Google Scholar

21. Reimann, S. M.; Manninen, M. Electronic structure of quantum dots. Rev. Mod. Phys. 2002, 74, 1283-1342, DOI: 10.1103/RevModPhys.74.1283 [Crossref], [CAS], Google Scholar 
22. Kimel, A. V. Ultrafast dynamics of the photo-induced magneto-optical Kerr effect in CdTe at room temperature. Phys. Rev. B 2000, 62, R10610- R10613, DOI: 10.1103/PhysRevB.62.R10610 [Crossref], [CAS], Google Scholar

23. Basov, D. N.; Averitt, R. D.; van der Marel, D.; Dressel, M.; Haule, K. Electrodynamics of correlated electron materials. Rev. Mod. Phys. 2011, 83, 471- 541, DOI: 10.1103/RevModPhys.83.471 [Crossref], [CAS], Google Scholar

24. Bedeaux, D.; Bloembergen, N. On the relation between macroscopic and microscopic nonlinear susceptibilities. Physica 1973, 69, 57- 66, DOI: 10.1016/0031-8914(73)90200-0 [Crossref], Google $\underline{\text { Scholar }}$

25. Sepúlveda, B.; González-Díaz, J. B.; García-Martín, A.; Lechuga, L. M.; Armelles, G. Plasmon-Induced Magneto-Optical Activity in Nanosized Gold Disks. Phys. Rev. Lett. 2010, 104, 147401 DOI: 10.1103/PhysRevLett.104.147401 [Crossref], [PubMed], [CAS], Google Scholar

26. Armelles, G.; Cebollada, A.; García-Martín, A.; González, M. U. Magnetoplasmonics: Combining Magnetic and Plasmonic Functionalities. Adv. Opt. Mater. 2013, 1, 10- 35, DOI: 10.1002/adom.201200011 [Crossref], Google Scholar

27. Moocarme, M.; Domínguez-Juárez, J. L.; Vuong, L. T. Ultralow-intensity magneto-optical and mechanical effects in metal nanocolloids. Nano Lett. 2014, 14, 1178- 1183, DOI: 10.1021/nl4039357 [ACS Full Text ], [CAS], Google Scholar

28. Singh, N. D.; Moocarme, M.; Edelstein, B.; Punnoose, N.; Vuong, L. T. Anomalously-large photoinduced magnetic response of metallic nanocolloids in aqueous solution using a solar simulator. Opt. Express 2012, 20, 19214, DOI: 10.1364/OE.20.019214 [Crossref], [PubMed], [CAS], Google Scholar

29. Hurst, J.; Oppeneer, P. M.; Manfredi, G.; Hervieux, P. A. Magnetic moment generation in small gold nanoparticles via the plasmonic inverse Faraday effect. Phys. Rev. B 2018, 98, 134439 DOI: 10.1103/PhysRevB.98.134439 [Crossref], [CAS], Google Scholar

30. Cheng, O. H. C.; Son, D. H.; Sheldon, M. Light-induced magnetism in plasmonic gold nanoparticles. Nat. Photonics 2020, 14, 365- 368, DOI: 10.1038/s41566-020-0603-3 [Crossref], [CAS], Google Scholar 31. Weick, G.; Weinmann, D. Lifetime of the surface magnetoplasmons in metallic nanoparticles. Phys. Rev. B 2011, 83, 125405 DOI: 10.1103/PhysRevB.83.125405 [Crossref], [CAS], Google Scholar

32. Zaitoun, M. A.; Mason, W. R.; Lin, C. T. Magnetic circular dichroism spectra for colloidal gold nanoparticles in xerogels at 5.5 K. J. Phys. Chem. B 2001, 105, 6780- 6784, DOI: 10.1021/jp003978r [ACS Full Text], [CAS], Google Scholar

33. Pineider, F. Circular Magnetoplasmonic Modes in Gold Nanoparticles. Nano Lett. 2013, 13, 47854789, DOI: 10.1021/n1402394p [ACS Full Text ], [CAS], Google Scholar

34. Mason, W. R. A Practical Guide to Magnetic Circular Dichroism Spectroscopy; John Wiley \& Sons, Inc, 2007.[Crossref], Google Scholar

35. Pakdel, S.; Miri, M. Faraday rotation and circular dichroism spectra of gold and silver nanoparticle aggregates. Phys. Rev. B 2012, 86, 235445 DOI: 10.1103/PhysRevB.86.235445 [Crossref], [CAS], Google Scholar

36. Shiratsu, T.; Yao, H. Size dependence of magneto-optical activity in silver nanoparticles with dimensions between 10 and $60 \mathrm{~nm}$ studied by MCD spectroscopy. Phys. Chem. Chem. Phys. 2018, 20, 4269- 4276, DOI: 10.1039/C7CP07695A [Crossref], [PubMed], [CAS], Google Scholar

37. Shiratsu, T.; Yao, H. Magnetic circular dichroism (MCD) in silver nanocubes with different sizes. Chem. Phys. Lett. 2018, 706, 607- 612, DOI: 10.1016/j.cplett.2018.07.015 [Crossref], [CAS], Google $\underline{\text { Scholar }}$

38. Han, B. Geometry-Modulated Magnetoplasmonic Optical Activity of Au Nanorod-Based Nanostructures. Nano Lett. 2017, 17, 6083- 6089, DOI: 10.1021/acs.nanolett.7b02583 [ACS Full Text ], [CAS], Google Scholar

39. Yao, H.; Shiratsu, T. Individual and collective modes of surface magnetoplasmon in thiolateprotected silver nanoparticles studied by MCD spectroscopy. Nanoscale 2016, 8, 11264-11274, DOI: 10.1039/C6NR00631K [Crossref], [PubMed], [CAS], Google Scholar 
40. Kataja, M.; Pourjamal, S.; van Dijken, S. Magnetic circular dichroism of non-local surface lattice resonances in magnetic nanoparticle arrays. Opt. Express 2016, 24, 3562, DOI: 10.1364/OE.24.003562 [Crossref], [PubMed], [CAS], Google Scholar

41. Jacobs, R. L. The electronic structure of the noble metals I. The energy bands. J. Phys. C: Solid State Phys. 1968, 1, 318, DOI: 10.1088/0022-3719/1/5/318 [Crossref], Google Scholar

42. Eckardt, H.; Fritsche, L.; Noffke, J. Self-consistent relativistic band structure of the noble metals. J. Phys. F: Met. Phys. 1984, 14, 97- 112, DOI: 10.1088/0305-4608/14/1/013 [Crossref], [CAS], Google $\underline{\text { Scholar }}$

43. Zhukov, V. P.; Aryasetiawan, F.; Chulkov, E. V.; Gurtubay, I. G. de.; Echenique, P. M. Corrected localdensity approximation band structures, linear-response dielectric functions, and quasiparticle lifetimes in noble metals. Phys. Rev. B 2001, 64, 195122 DOI: 10.1103/PhysRevB.64.195122 [Crossref], [CAS], Google Scholar

44. Moores, A.; Goettmann, F. The plasmon band in noble metal nanoparticles: an introduction to theory and applications. New J. Chem. 2006, 30, 1121, DOI: 10.1039/b604038c [Crossref], [CAS], Google Scholar

45. Guerrisi, M.; Rosei, R.; Winsemius, P. Splitting of the interband absorption edge in Au. Phys. Rev. B 1975, 12, 557- 563, DOI: 10.1103/PhysRevB.12.557 [Crossref], [CAS], Google Scholar

46. Winsemius, P.; Guerrisi, M.; Rosei, R. Splitting of the interband absorption edge in Au: Temperature dependence. Phys. Rev. B 1975, 12, 4570- 4572, DOI: 10.1103/PhysRevB.12.4570 [Crossref], [CAS], Google Scholar

47. Yang, H. U. Optical dielectric function of silver. Phys. Rev. B 2015, 91, 235137 DOI: 10.1103/PhysRevB.91.235137 [Crossref], [CAS], Google Scholar

48. Stahrenberg, K. Optical properties of copper and silver in the energy range 2.5-9.0 eV. Phys. Rev. B 2001, 64, 115111 DOI: 10.1103/PhysRevB.64.115111 [Crossref], [CAS], Google Scholar

49. Christensen, N. E.; Seraphin, B. O. Relativistic Band Calculation and the Optical Properties of Gold. Phys. Rev. B 1971, 4, 3321-3344, DOI: 10.1103/PhysRevB.4.3321 [Crossref], Google Scholar

50. Rangel, T. Band structure of gold from many-body perturbation theory. Phys. Rev. B 2012, 86, 125125 DOI: 10.1103/PhysRevB.86.125125 [Crossref], [CAS], Google Scholar

51. Alkauskas, A.; Schneider, S. D.; Hébert, C.; Sagmeister, S.; Draxl, C. Dynamic structure factors of Cu, $\mathrm{Ag}$, and Au: Comparative study from first principles. Phys. Rev. B 2013, 88, 195124 DOI: 10.1103/PhysRevB.88.195124 [Crossref], [CAS], Google Scholar

52. Uba, L.; Uba, S.; Antonov, V. N. Magneto-optical Kerr spectroscopy of noble metals. Phys. Rev. B 2017, 96, 235132 DOI: 10.1103/PhysRevB.96.235132 [Crossref], [CAS], Google Scholar

53. Cretu, C.; van der Lingen, E. Coloured gold alloys. Gold Bull. 1999, 32, 115- 126, DOI: 10.1007/BF03214796 [Crossref], [CAS], Google Scholar

54. Camley, R. E.; Mills, D. L. Collective excitations of semi-infinite superlattice structures: Surface plasmons, bulk plasmons, and the electron-energy-loss spectrum. Phys. Rev. B 1984, 29, 16951706, DOI: 10.1103/PhysRevB.29.1695 [Crossref], [CAS], Google Scholar

55. Grésillon, S. Experimental Observation of Localized Optical Excitations in Random Metal-Dielectric Films. Phys. Rev. Lett. 1999, 82, 4520- 4523, DOI: 10.1103/PhysRevLett.82.4520 [Crossref], [CAS], Google Scholar

56. Kelly, K. L.; Coronado, E.; Zhao, L. L.; Schatz, G. C. The optical properties of metal nanoparticles: The influence of size, shape, and dielectric environment. J. Phys. Chem. B 2003, 107, 668-677, DOI: 10.1021/jp026731y [ACS Full Text ], [CAS], Google Scholar

57. Beversluis, M. R.; Bouhelier, A.; Novotny, L. Continuum generation from single gold nanostructures through near-field mediated intraband transitions. Phys. Rev. B 2003, 68, 115433 DOI: 10.1103/PhysRevB.68.115433 [Crossref], [CAS], Google Scholar

58. Rycenga, M. Controlling the synthesis and assembly of silver nanostructures for plasmonic applications. Chem. Rev. 2011, 111, 3669- 3712, DOI: 10.1021/cr100275d [ACS Full Text], [CAS], Google Scholar 
59. Wysin, G. M.; Chikan, V.; Young, N.; Dani, R. K. Effects of interband transitions on Faraday rotation in metallic nanoparticles. J. Phys.: Condens. Matter 2013, 25, 325302 DOI: 10.1088/09538984/25/32/325302 [Crossref], [PubMed], [CAS], Google Scholar

60. Su, K. H. Interparticle coupling effects on plasmon resonances of nanogold particles. Nano Lett. 2003, 3, 1087- 1090, DOI: 10.1021/nl034197f [ACS Full Text ], [CAS], Google Scholar

61. Weick, G.; Mariani, E. Tunable plasmon polaritons in arrays of interacting metallic nanoparticles. Eur. Phys. J. B 2015, 88, 1-8, DOI: 10.1140/epjb/e2014-50658-2 [Crossref], [CAS], Google Scholar

62. Garnett, J. C. M., XII. Colours in metal glasses and in metallic films. Philos. Trans. R. Soc., A 1904, 203, 385-420[Crossref], [CAS], Google Scholar

63. Markel, V. A. Introduction to the Maxwell Garnett approximation: tutorial. J. Opt. Soc. Am. A 2016, 33, 1244, DOI: 10.1364/JOSAA.33.001244 [Crossref], [CAS], Google Scholar

64. Bruggeman, D. A. G. Berechnung verschiedener physikalischer Konstanten von heterogenen Substanzen. I. Dielektrizitätskonstanten und Leitfähigkeiten der Mischkörper aus isotropen Substanzen. Ann. Phys. 1935, 416, 636- 664, DOI: 10.1002/andp.19354160705 [Crossref], Google $\underline{\text { Scholar }}$

65. Niklasson, G. A.; Granqvist, C. G.; Hunderi, O. Effective medium models for the optical properties of inhomogeneous materials. Appl. Opt. 1981, 20, 26, DOI: 10.1364/AO.20.000026 [Crossref], [PubMed], [CAS], Google Scholar

66. Koledintseva, M. Y.; DuBroff, R. E.; Schwartz, R. W. A Maxwell Garnett model for dielectric mixtures containing conducting particles at optical frequencies. Prog. Electromagn. Res. 2006, 63, 223242, DOI: 10.2528/PIER06052601 [Crossref], Google Scholar

67. Liu, Y. Two dimensional dipolar coupling in monolayers of silver and gold nanoparticles on a dielectric substrate. Nanoscale 2014, 6, 12080- 12088, DOI: 10.1039/C4NR03292F [Crossref], [PubMed], [CAS], Google Scholar

68. Voigt, W. Magneto- und elektrooptik Publ. 1908 Leipzig by B G Teubner 1908.Google Scholar 69. Maillard, M.; Giorgio, S.; Pileni, M.-P. Silver Nanodisks. Adv. Mater. 2002, 14, 1084- 1086, DOI: 10.1002/1521-4095(20020805)14:15<1084::AID-ADMA1084>3.0.CO;2-L [Crossref], [CAS], Google Scholar

70. Chen, S.; Fan, Z.; Carroll, D. L. Silver nanodisks: Synthesis, characterization, and self-assembly. J. Phys. Chem. B 2002, 106, 10777- 10781, DOI: 10.1021/jp026376b [ACS Full Text], [CAS], Google $\underline{\text { Scholar }}$

71. Tsuji, M. Rapid Transformation from Spherical Nanoparticles, Nanorods, Cubes, or Bipyramids to Triangular Prisms of Silver with PVP, Citrate, and $\mathrm{H}_{2} \mathrm{O}_{2}$. Langmuir 2012, 28, 8845- 8861, DOI: 10.1021/la3001027 [ACS Full Text], [CAS], Google Scholar

72. Mulvihill, M. J.; Ling, X. Y.; Henzie, J.; Yang, P. Anisotropic Etching of Silver Nanoparticles for Plasmonic Structures Capable of Single-Particle SERS. J. Am. Chem. Soc. 2010, 132, 268- 274, DOI: 10.1021/ja906954f [ACS Full Text ], [CAS], Google Scholar

73. Marini, A.; Del Sole, R.; Onida, G. First-principles calculation of the plasmon resonance and of the reflectance spectrum of silver in the GW approximation. Phys. Rev. B 2002, 66, 115101 DOI: 10.1103/PhysRevB.66.115101 [Crossref], [CAS], Google Scholar 